\title{
Подвижность электронов в инверсионных слоях полностью обедняемых пленок кремний-на-изоляторе
}

\author{
(C) Э.Г. Зайцева, О.В. Наумова, Б.И. Фомин
}

Институт ффизики полупроводников им. А.В. Ржанова Сибирского отделения Российской академии наук, 630090 Новосибирск, Россия

E-mail: ZaytsevaElza@yandex.ru

(Получена 13 июля 2016 г. Принята к печати 1 августа 2016 г.)

Исследована подвижность электронов $\mu_{\text {eff }}$ в инверсионных слоях двухзатворных полностью обедняемых КНИ (кремний-на-изоляторе) МОП транзисторов в зависимости от плотности индуцированных носителей заряда $N_{e}$ и температуры $T$ при разных режимах пленки КНИ со стороны одного из затворов (инверсия-обогащение). Показано, что при большой плотности индуцированных носителей заряда $\left(N_{e}>6 \cdot 10^{12} \mathrm{~cm}^{-2}\right)$ зависимости $\mu_{\mathrm{eff}}(T)$ позволяют выделить компоненты подвижности $\mu_{\mathrm{eff}}$, связанные с рассеянием на поверхностных фононах и микрорельефе границы раздела пленка/диэлектрик. Зависимости $\mu_{\mathrm{eff}}\left(N_{e}\right)$ могут быть аппроксимированы степенными функциями $\mu_{\mathrm{eff}}\left(N_{e}\right) \propto N_{e}^{-n}$. Определены значения показателей $n$ зависимостей и доминирующие механизмы рассеяния электронов, индуцированных вблизи границы раздела пленки КНИ со скрытым диэлектриком, для различных интервалов $N_{e}$ и режимов пленки со стороны поверхности.

DOI: $10.21883 /$ FTP.2017.04.44334.8369

\section{1. Введение}

Полностью обедняемые пленки являются основой современной микро- и наноэлектроники. Любая пленка имеет две границы раздела (ГР) с окружающими ее диэлектриками - c диэлектриком, на котором пленка размещена (как правило, этот диэлектрик отсекает пленку от проводящей подложки), и с диэлектриком на ее поверхности. Фундаментальной особенностью тонкопленочных структур является взаимосвязь потенциалов противоположных ГР пленка/диэлектрик, или так называемый coupling-эффект [1-4].

Вследствие coupling-эффекта электрические параметры тонкопленочных структур (и приборов на их основе) определяются потенциалами на обеих ГР пленка/диэлектрик $[3,4]$. Поэтому свойства обеих ГР пленок становятся приборно-значимыми.

Одним из основных параметров МОП транзисторов (структуры металл-окисел-полупроводник) и параметром, который характеризует свойства системы полупроводник/диэлектрик, является подвижность носителей заряда. Вблизи гетерограниц она определяется несколькими механизмами рассеяния: кулоновским рассеянием, рассеянием на поверхностных и объемных фононах, рассеянием на микрорельефе ГР [5-7].

Хорошо известно, что для носителей заряда в инверсионных слоях МОП транзисторов на объемном кремнии характерна универсальная зависимость подвижности от эффективного поля $E_{\text {eff }}$ в канале транзистора, направленного перпендикулярно плоскости индуцированного канала проводимости $[8,9]$. Значения $E_{\text {eff }}$ определяют распределение носителей заряда относительно гетерограницы, поэтому существует однозначная связь между $E_{\text {eff }}$ и доминирующим механизмом рассеяния носителей заряда. Установлено, что в области сла- бых полей, $E_{\text {eff }}<2 \cdot 10^{5} \mathrm{~B} / \mathrm{cm}$, доминирует рассеяние на поверхностных состояниях. В области средних полей,

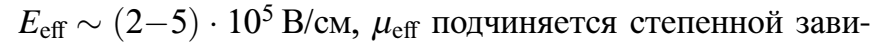
симости $\mu_{\mathrm{eff}} \propto E_{\mathrm{eff}}^{-n}$ с показателем $n=0.3$ и определяется рассеянием на фононах. В области сильных полей доминирует рассеяние на микрорельефе ГР. Однако в МОП транзисторах на основе полностью обедняемых пленок КНИ вследствие coupling-эффекта теряется универсальность зависимостей $\mu_{\text {eff }}\left(E_{\text {eff }}\right)$. Для таких структур наблюдается целая серия зависимостей $\mu_{\mathrm{eff}}\left(E_{\mathrm{eff}}\right)$, где значения подвижности носителей заряда определяются не только потенциалом на ГР, вблизи которой индуцируется канал проводимости, но и потенциалом на противоположной гетерогранице [10]. В условиях couplingэффекта одно и то же значение $E_{\text {eff }}$ может быть создано разной комбинацией потенциалов на гетерограницах и соответствовать разному распределению носителей заряда в пленке относительно ГР [11].

В работе [10] была предложена замена полевой зависимости подвижности зависимостью $\mu_{\text {eff }}$ от плотности индуцированных носителей заряда $N_{e}$ для определения диапазона возможных значений подвижности и доминирующих механизмов рассеяния в тонкопленочных структурах. Было показано, что для электронов в обогащении зависимости $\mu_{\mathrm{eff}}\left(N_{e}\right)$ могут быть аппроксимированы степенными функциями $\mu_{\mathrm{eff}}\left(N_{e}\right) \propto N_{e}^{-n}$, где показатель $n$ зависит от значений $N_{e}$ и режима пленки со стороны поверхности и, как в полевой зависимости подвижности, определяется механизмом рассеяния носителей заряда. Однако наиболее полные исследования полевых зависимостей подвижности с идентификацией механизмов рассеяния были проведены и получены для носителей в инверсионных слоях на объемном кремнии (в стандартных режимах МОП транзисторов на объемном материале) $[5,6,8,9,12]$. 


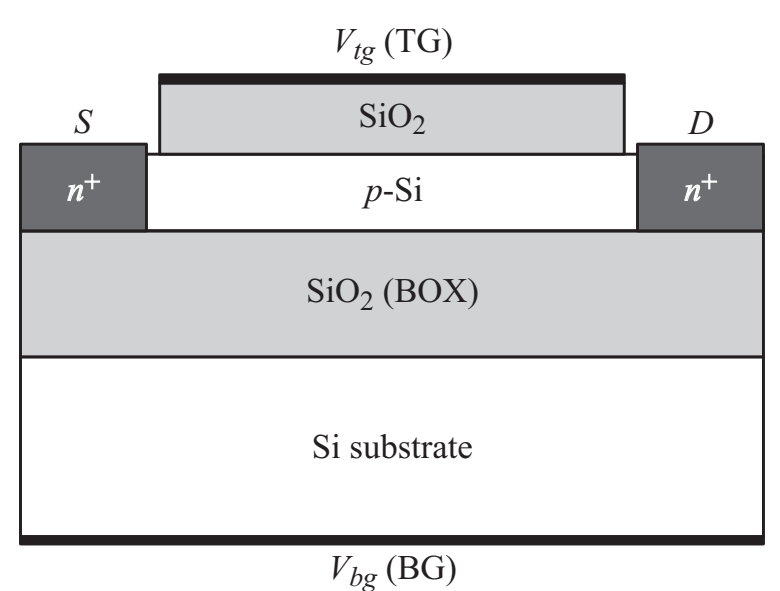

Рис. 1. Схематическое изображение сечения полностью обедняемого КНИ МОП транзистора. $S-$ исток, $D-$ сток.

Целью данной работы являлось исследование поведения зависимостей $\mu_{\mathrm{eff}}\left(N_{e}\right)$ и определение доминирующих механизмов рассеяния электронов в инверсионных слоях в полностью обедняемых пленках КНИ при различных режимах (инверсия-обогащение) пленок со стороны одной из поверхностей. Для этого в работе использовались $n$-канальные полностью обедняемые КНИ МОП транзисторы. Напряжением на одном из затворов (BG, в качестве которого использовалась подложка структур КНИ) формировался электронный канал проводимости вблизи границы раздела $\mathrm{Si}$ со скрытым диэлектриком. Напряжением на другом затворе (TG) менялся режим слоя КНИ со стороны затвора TG от обогащения до инверсии при измерении затворных зависимостей $I_{d s}\left(V_{b g}\right)$ транзисторов в диапазоне температур $T=78-300 \mathrm{~K}$

\section{2. Экспериментальные данные и методика}

В работе использовались $n$-канальные КНИ МОП транзисторы. Транзисторы были изготовлены на слоях КНИ $p$-типа проводимости с концентрацией акцепторов $N_{a}=2 \cdot 10^{16} \mathrm{~cm}^{-3}$. Структуры КНИ были изготовлены методом Smart-Cut, получившим коммерческое распространение. Толщина отсеченного слоя кремния составляла 30 нм, толщина скрытого окисла (buried oxide $\mathrm{BOX)} t_{\mathrm{BOX}}=200$ нм. Толщина подзатворного диэлектрика транзисторов составляла $t_{\text {ох }}=250 \mathrm{Hм}$, длина канала $L=10$ мкм, ширина $W=3$ мкм. Схематическое изображение КНИ МОП транзистора приведено на рис. 1. Детали метода изготовления КНИ транзисторов описаны в работе [13].

Измерялись зависимости тока стока $I_{d s}$ от напряжения на затворе BG $\left(V_{b g}\right)$ при фиксированном напряжении на затворе TG. Тянущее напряжение $V_{d s}$ составляло 100 мВ. Измерения проводились при температурах в диапазоне 78-300 K. Напряжение на затворе TG варьировалось в диапазоне $(-50)-50 \mathrm{~B}$ с шагом $5 \mathrm{~B}$.

Из полученных зависимостей $I_{d s}\left(V_{b g}\right)$ рассчитывались пороговое напряжение $V_{\mathrm{th}}$, подвижность электронов $\mu_{\text {eff }}$ и плотность электронов в канале транзистора $N_{e}$. На основе рассчитанных данных строились зависимости $V_{\text {th }}\left(V_{t g}\right), \mu_{\text {eff }}\left(N_{e}\right)$ и $\mu_{\text {eff }}(T)$.

Значения эффективной подвижности электронов рассчитывались по методике, изложенной в работах $[8,14]$ :

$$
\mu_{\mathrm{eff}}=\frac{I_{d s}}{(W / L) C_{\mathrm{BOX}} V_{d s}\left(V_{b g}-V_{\mathrm{th}}\right)},
$$

где $C_{\text {ВOX }}$ - емкость скрытого окисла структур КНИ.

Пороговое напряжение $V_{\text {th }}$ определялось методом экстраполяции функции $I_{d s} /\left(g_{m}\right)^{1 / 2}$ (где $\left.g_{m}=d I_{d s} / d V_{b g}\right)$ на ось напряжений $V_{b g}[15]$.

Плотность электронов, индуцированных в канале транзистора, определялась как

$$
N_{e}=\frac{C_{\mathrm{BOX}}}{q}\left(V_{b g}-V_{\mathrm{th}}\right),
$$

$q-$ заряд электрона.

\section{3. Результаты}

На рис. 2 (вставка) представлены типичные зависимости $I_{d s}\left(V_{b g}\right)$, измеренные при разных напряжениях на затворе TG, и соответствующая им зависимость порогового напряжения $V_{\text {th }}$ от напряжения $V_{t g}$ для исследуемых КНИ МОП транзисторов. Видно, что при изменении напряжения $V_{t g}$ происходит сдвиг характеристик $I_{d s}\left(V_{b g}\right)$ но оси напряжений соответственно и изменение порогового напряжения $V_{\mathrm{th}}$. На зависимости $V_{\mathrm{th}}\left(V_{t g}\right)$ можно выделить три характерных участка — две

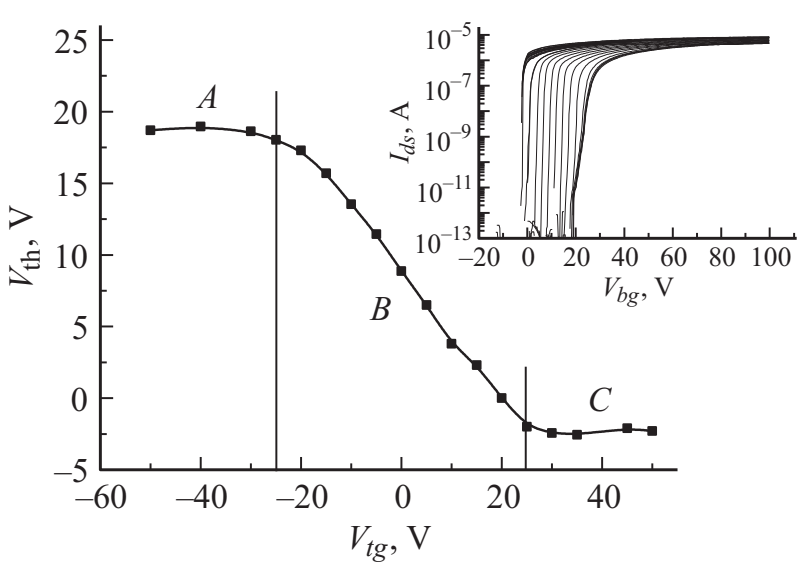

Рис. 2. Зависимости $V_{\text {th }}\left(V_{t g}\right)$ и $I_{d s}\left(V_{b g}\right)$ (на вставке) для $n$-канальных КНИ МОП транзисторов, измеренные при значениях $V_{t g}$ в диапазоне от -50 до $50 \mathrm{~B}$ с шагом $5 \mathrm{~B}$, при $V_{d s}=0.1 \mathrm{~B}, T=300 \mathrm{~K}$. Область $A$ на зависимости $V_{\text {th }}\left(V_{t g}\right)$ соответствует обогащению, область $C$ - инверсии, линейная зависимость в переходной области $B-$ обеднению пленки $p$ - $\mathrm{Si}$ со стороны TG. 


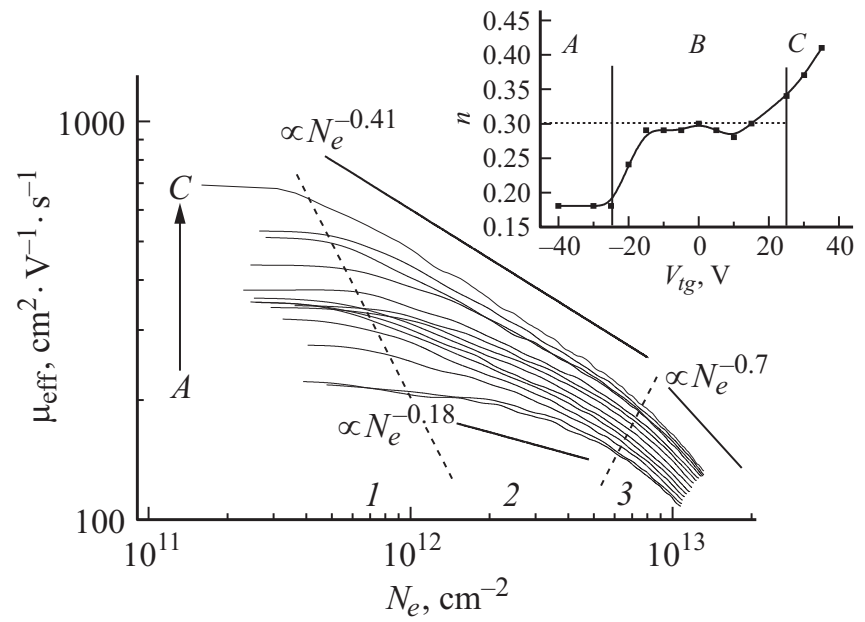

Рис. 3. Зависимости $\mu_{\mathrm{eff}}\left(N_{e}\right)$ и аппроксимации $\mu_{\mathrm{eff}} \propto N_{e}^{-n}$ для КНИ МОП транзисторов при изменении режима слоя КНИ со стороны TG от обогащения $(A)$ до инверсии $(C)$. На вставке зависимость показателя $n$ от напряжения $V_{t g}$ в области 2 .

области насыщения $(A, C)$ и переходную область $B$. Два плато на зависимости $V_{\mathrm{th}}\left(V_{t g}\right)$ (области насыщения $A$ и $C$ ) соответствуют состояниям экранирования - coстоянию обогащения или сильной инверсии пленки $p$-Si со стороны затвора TG при $V_{t g}<-20$ В и $V_{t g}>20 \mathrm{~B}$ соответственно. Таким образом, смещение зависимостей $I_{d s}\left(V_{b g}\right)$ влево по оси напряжений на рис. 2, или уменьшение $V_{\text {th }}$ с увеличением $V_{t g}$, наблюдается при изменении состояния приповерхностного слоя $p$-Si от обогащения до инверсии. Линейный участок в переходной области $B$ при $V_{t g}$ в диапазоне $(-15,15)$ В соответствует полному обеднению пленки КНИ со стороны TG $[10,16]$.

На рис. 3 представлены зависимости эффективной подвижности электронов $\mu_{\text {eff }}$, индуцированных напряжением на затворе BG, от их плотности $N_{e}$, рассчитанные на основе полученных характеристик $I_{d s}\left(V_{b g}\right)$. Видно, что подвижность электронов изменяется в диапазоне $690-110 \mathrm{~cm}^{2} \cdot \mathrm{B}^{-1} \cdot \mathrm{c}^{-1}$ при изменении $N_{e}$ в пределах $2 \cdot 10^{11}-1.2 \cdot 10^{13} \cdot \mathrm{cm}^{-2}$ и увеличивается при переходе пленки от режима $A$ к режиму $C$ (при увеличении положительного напряжения на TG). Аналогично подвижности электронов в аккумуляции [10] или полевой зависимости подвижности в МОП транзисторах на объемном кремнии $[8,12]$ зависимости $\mu_{\text {eff }}\left(N_{e}\right)$ для электронов в инверсии могут быть аппроксимированы степенными функциями $\propto N_{e}^{-n}$. Аппроксимация зависимостей $\mu_{\text {eff }}\left(N_{e}\right)$ степенными функциями $\mu_{\text {eff }} \propto N_{e}^{-n}$ позволяет выделить три интервала $N_{e}$ с характерными значениями $n$ (отделены штриховыми линиями на рис. 3). В области 1 , при $N_{e}=2 \cdot 10^{11}-10^{12} \mathrm{~cm}^{-2}$, и в области 3 , при $N_{e}>(6-7) \cdot 10^{12} \mathrm{~cm}^{-2}$, значения $n$ не зависят от $V_{t g}$ и составляют 0 и 0.7 соответственно. В области 2 , при $N_{e}=(1-6) \cdot 10^{12} \mathrm{~cm}^{-2}$, значения $n$ увеличиваются с увеличением напряжения $V_{t g}$. Зависимость $n\left(V_{t g}\right)$ для области 2 показана на вставке к рис. 3. Видно, что при значениях $V_{t g}$ в диапазоне $(-15,15)$ В, т.е. в режиме $B$, когда слой $\mathrm{Si}$ со стороны TG полностью обеднен (см. рис. 2), показатель степени $n$ имеет постоянное значение $\sim 0.3$.

В работе [10] показано, что в тонких пленках закономерен переход от полевой зависимости подвижности $\mu_{\mathrm{eff}}\left(E_{\mathrm{eff}}\right)$ к зависимости подвижности от плотности индуцированных носителей заряда $\mu_{\mathrm{eff}}\left(N_{e}\right)$. Хорошо известно, что в МОП транзисторах на объемном кремнии показатель $n=0.3$ зависимости $\mu_{\mathrm{eff}} \propto E_{\mathrm{eff}}^{-n}$ в области средних значений поля $E_{\mathrm{eff}}=(2-5) \cdot 10^{5} \mathrm{~B} / \mathrm{cm}$ определяется рассеянием на фононах с температурной зависимостью подвижности $\mu_{\mathrm{eff}} \propto T^{-1.75}[8,12]$.

На рис. 4 представлены типичные температурные зависимости подвижности электронов в инверсии в области средних и высоких значений $N_{e}$, построенные в координатах $\mu_{\mathrm{eff}}\left(T^{-1.75}\right)$. Показаны результаты измерений при $N_{e}=2 \cdot 10^{12}$ и $10^{13} \mathrm{~cm}^{-2}$ (в интервалах 2 и 3 на рис. 3 соответственно) для режимов $A, B$ и $C$ пленки со стороны TG (при напряжениях $V_{t g}=-40,0,35 \mathrm{~B}$ соответственно, см. рис. 2), т.е. для режимов пленки с разными показателями $n$. Для сравнения на вставках к
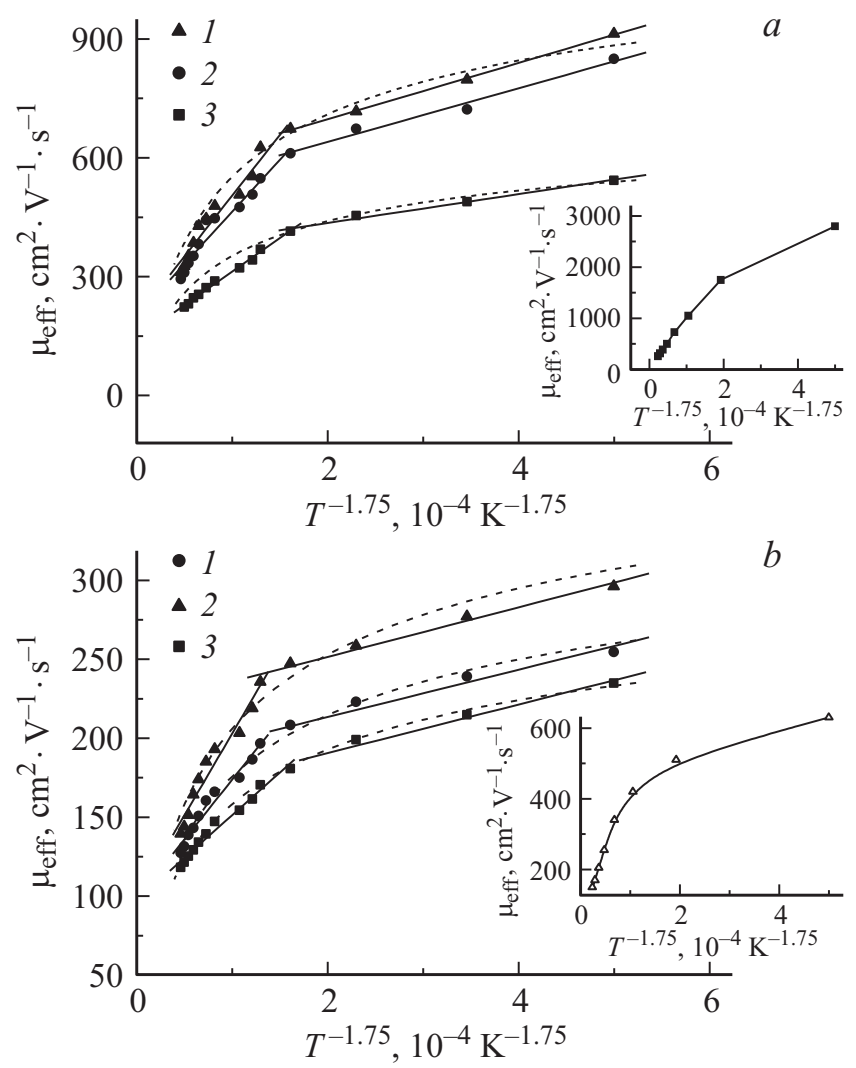

Рис. 4. Зависимости $\mu_{\mathrm{eff}}\left(T^{-1.75}\right): a-$ область 2, $N_{e}=2 \cdot 10^{12} \mathrm{~cm}^{-2} ; b$ - область $3, N_{e}=10^{13} \mathrm{~cm}^{-2}$. Режим слоя КНИ со стороны ТG: 1 - обогащение $(A), 2$ - обеднение $(B), 3$ - инверсия $(C)$. Точки - экспериментальные значения, сплошные линии - их линейная аппроксимация, штриховые линии - аппроксимация по формуле (5). На вставках зависимости $\mu_{\mathrm{eff}}\left(T^{-1.75}\right)$ для МОП транзисторов на объемном Si при $E_{\text {eff }}=3 \cdot 10^{5}(a)$ и $10^{6} \mathrm{~B} / \mathrm{cm}(b)$. 
Значения коэффициентов $B_{1}$ и $B_{2}$ для зависимостей $\mu_{\mathrm{eff}}\left(T^{-1.75}\right)$ в полностью обедняемых МОП транзисторах при разной плотности электронов $N_{e}$ и режимах слоя КНИ со стороны ТG

\begin{tabular}{|c|c|c|c|c|c|c|}
\hline \multirow{2}{*}{ Режим измерений } & \multicolumn{3}{|c|}{$N_{e}=2 \cdot 10^{12} \mathrm{~cm}^{-2}$ (область 2) } & \multicolumn{3}{|c|}{$N_{e}=10^{13} \mathrm{~cm}^{-2}$ (область 3$)$} \\
\hline & $A$ & $B$ & $C$ & $A$ & $B$ & $C$ \\
\hline Показатель $n$ & 0.1 & 0.3 & 0.37 & \multicolumn{3}{|c|}{0.7} \\
\hline $\begin{array}{l}B_{1} \\
B_{2}\end{array}$ & $\begin{array}{l}1.7 \cdot 10^{6} \\
3.6 \cdot 10^{5}\end{array}$ & $\begin{array}{l}2.8 \cdot 10^{6} \\
6.8 \cdot 10^{5}\end{array}$ & $\begin{array}{l}3.3 \cdot 10^{6} \\
7.1 \cdot 10^{5}\end{array}$ & $\begin{array}{l}5.8 \cdot 10^{5} \\
1.5 \cdot 10^{5}\end{array}$ & $\begin{array}{l}7.6 \cdot 10^{5} \\
1.5 \cdot 10^{5}\end{array}$ & $\begin{array}{l}1.0 \cdot 10^{6} \\
1.5 \cdot 10^{5}\end{array}$ \\
\hline
\end{tabular}

Примечание. $A$ - обогащение, $B$ - обеднение, $C$ - инверсия.

рис. 4 показаны зависимости $\mu_{\mathrm{eff}}\left(T^{-1.75}\right)$ для электронов в инверсионном слое МОП транзисторов на объемном кремнии, построенные по данным работы [8] в области средних и высоких значений $E_{\text {eff }}$ - при $E_{\text {eff }}=3 \cdot 10^{5}$ и $10^{6} \mathrm{~B} / \mathrm{cm}$ соответственно. Видно, что в транзисторах как на полностью обедняемых слоях КНИ, так и на объемном кремнии для зависимостей $\mu_{\mathrm{eff}}\left(T^{-1.75}\right)$ характерны два линейных участка с наклонами $B_{1}$ в диапазоне температур $T \approx 300-180 \mathrm{~K}$ и $B_{2}$ при более низких температурах. Режимы измерений, значения показателей $n$ и коэффициентов $B_{1}$ и $B_{2}$ представлены в таблице.

Из таблицы и рис. 4 видно, что независимо от режимов измерений выполняется условие $B_{1}>B_{2}$. При переходе от режима обогащения $(A)$ к режиму инверсии $(C)$ пленки со стороны TG наблюдается увеличение коэффициентов $B_{i}$. Исключение составляют режимы $3 A, 3 B$ и $3 C$ в диапазоне температур $78-180 \mathrm{~K}$, где наблюдается практически параллельный сдвиг зависимостей $\mu_{\text {eff }}\left(T^{-1.75}\right)$ по оси $\mu_{\text {eff }}$, т.еं. $B_{2} \approx$ const при изменении режима пленки со стороны TG (рис. $4, b)$.

\section{4. Обсуждение результатов}

Таким образом, результаты показывают (рис. 3), что подвижность электронов в инверсионных слоях, как и в слоях обогащения (см. [10]) полностью обедняемых КНИ транзисторов, может быть описана степенной функцией от $N_{e}$ по аналогии с полевой зависимостью подвижности электронов в инверсионных слоях МОП транзисторов на объемном кремнии.

Значения $\mu_{\text {eff }}$ и показателей $n$ зависят от напряжений на обоих затворах КНИ транзистора, которые определяют плотность и распределение носителей (положение центроида заряда) по сечению пленки [10]. Понятно, что при больших отрицательных напряжениях на TG (в режиме $A$ ) электроны максимально смещены к границе раздела КНИ/ВОХ. При больших положительных напряжениях на TG (в режиме $C$ ) электроны максимально смещены к границе раздела КНИ/оксид, по направлению к TG. Результаты показывают (рис. 3), что при $T=300 \mathrm{~K}$ для $N_{e}$ в интервалах 1 и 3 значения степенных показателей $n$ зависимостей $\mu_{\mathrm{eff}} \propto N_{e}^{-n}$ различаются, но остаются постоянными в пределах каждого из этих интервалов при изменении $V_{t g}$ (изменении режима пленки со стороны TG). Это значит, что в областях 1 и 3, несмотря на изменение в положении центроида заряда, доминирующие механизмы рассеяния электронов сохраняются постоянными.

В режиме $2 B$ (области средних значений $N_{e}$ и обеднения пленки, см. таблицу) степенной показатель $n=0.3$ и температурные зависимости подвижности подчиняются зависимости $\mu_{\text {eff }} \propto T^{-1.75}$. Тот же показатель $n=0.3$ в полевой зависимости подвижности $\mu_{\text {eff }} \propto E_{\text {eff }}^{-n}$ и тот же ход температурных зависимостей $\mu_{\text {eff }} \propto T^{-1.75}$ наблюдаются в области средних значений $E_{\text {eff }}$ в МОП транзисторах на объемном кремнии [8].

В МОП транзисторах на объемном кремнии положение носителей заряда относительно границы раздела $\mathrm{Si} / \mathrm{SiO}_{2}$ и доминирующий механизм рассеяния соответственно определяются значением $E_{\text {eff. Для ин- }}$ версных носителей заряда в области слабых полей, $E_{\text {eff }}<2 \cdot 10^{5} \mathrm{~B} /$ см, доминирует кулоновское рассеяние, именно рассеяние на поверхностных состояниях. С увеличением плотности индуцированных носителей заряда в канале его вклад уменьшается, так что в области средних полей доминирующим становится рассеяние на фононах. В области сильных полей, при $E_{\mathrm{eff}}>5 \cdot 10^{5} \mathrm{~B} / \mathrm{cm}$, доминирует рассеяние на микрорельефе ГР. Поэтому по аналогии с зависимостями $\mu_{\mathrm{eff}}\left(E_{\mathrm{eff}}\right)$ для инверсных электронов в МОП транзисторах на объемном кремнии логично предположить, что в полностью обедняемых пленках КНИ в интервале 1 (малые значения $N_{e}$ или $\left.E_{\text {eff }}\right)$ для зависимостей $\mu_{\text {eff }} \propto N_{e}^{-n}$ с показателем $n=0$ доминирует кулоновское рассеяние на поверхностных состояниях, в интервале 2 при характерных значениях $n=0.3$ и зависимостях $\mu_{\text {eff }} \propto T^{-1.75}-$ рассеяние на фононах, и в интервале 3 (больших значений $N_{e}$ или $E_{\text {eff }}$ ) с $n=0.7$ доминирует рассеяние на микрорельефе ГР. Однако в полностью обедняемых пленках КНИ зависимости $\mu_{\text {eff }} \propto T^{-1.75}$ наблюдаются и при других режимах пленки, в интервалах 2 и 3 с другими значениями $n$ (см. рис. 4 и таблицу).

Как говорилось выше, подвижность носителей заряда является многокомпонентной функцией. Она определяется несколькими механизмами рассеяния: кулоновским - компонента $\mu_{C}$, рассеянием на фононах (объемных $-\mu_{V p h}$, поверхностных $\left.-\mu_{s p h}\right)$, рассеянием на микрорельефе ГР - $\mu_{s r}$. В тонких пленках для эффективной подвижности $\mu_{1}$ носителей заряда, индуци- 
рованных вблизи одной из ГР, добавляется компонента подвижности $\mu_{2}$, связанная с рассеянием на второй ГР пленки:

$$
\mu_{1}=\left(\frac{1}{\mu_{C}}+\frac{1}{\mu_{V p h}}+\frac{1}{\mu_{s p h}}+\frac{1}{\mu_{s r}}+\frac{1}{\mu_{2}}\right)^{-1} .
$$

Понятно, что при большой плотности индуцированных носителей заряда $N_{e}$ (в области 3, рис. 3), когда выполняется условие $V_{b g} \gg V_{t g}$, можно пренебречь первым и последним членами в выражении (3). Действительно, при большой плотности индуцированных напряжением $V_{b g}$ носителей заряда можно пренебречь кулоновским рассеянием на поверхностных состояниях. Большая плотность электронов в индуцированном вблизи ГР с ВОХ канале проводимости достигается при больших положительных напряжениях на BG. При этом при увеличении положительного напряжения на BG происходит смещение электронов по направлению к ВОХ, которое усиливается при подаче отрицательных напряжений на TG. Распределение электронов по сечению пленки при различных режимах со стороны TG показано, например, в работах [10,17]. Таким образом, при больших положительных напряжениях $V_{b g}$ и отрицательных $V_{t g}$ (в режиме $3 A$, инверсии-обогащения на противоположных сторонах пленки) электронный канал проводимости максимально приближен к внутренней ГР $\mathrm{Si} / \mathrm{SiO}_{2}$ (ВОХ). Понятно, что в режиме $3 A$ доля „объемных“ электронов минимальна, и вклад рассеяния на ионизованной примеси и объемных фононах также должен быть не существенным.

Рассеяние на объемных фононах является наиболее изученным механизмом рассеяния. Подвижность, ограниченная рассеянием на объемных фононах, $\mu_{V p h}$, зависит от типа и температуры материала и описывается функцией [5]

$$
\mu_{V p h}=\mu_{0}\left(\frac{T}{300}\right)^{\theta},
$$

где $\mu_{0}$ - максимальная подвижность носителей заряда в материале, $\theta$ - показатель степени, зависящий от полупроводникового материала и от типа носителей заряда. Для электронов в кремнии, согласно работам $[18,19]$, $\mu_{0}=1414 \mathrm{~cm}^{2} \cdot \mathrm{B}^{-1} \cdot \mathrm{c}^{-1}$, а значение показателя степени $\theta=2.285$.

Подвижность носителей заряда, ограниченная рассеянием на микрорельефе ГР, не зависит от температуры, $\mu_{s r} \neq f(T)=$ const [20-22]. Подвижность, ограниченная рассеянием на поверхностных фононах, согласно модели Ломбарди [5,7], обратно пропорциональна температуре, $\mu_{s p h}(T)=A+b T^{-1}$, где коэффициенты $a$ и $b$ зависят от напряженности эффективного поля в канале $\left(a \propto E_{\mathrm{eff}}^{-1}\right.$ и $b \propto E_{\mathrm{eff}}^{-1 / 3}$ ). В области больших полей (при больших $N_{e}$ соответственно) $\mu_{s p h}(T)$ может быть аппроксимирована зависимостью $\mu_{s p h}(T)=b T^{-1}$. Таким образом, при фиксированном поле (при фиксированных значениях $N_{e}$ ) в

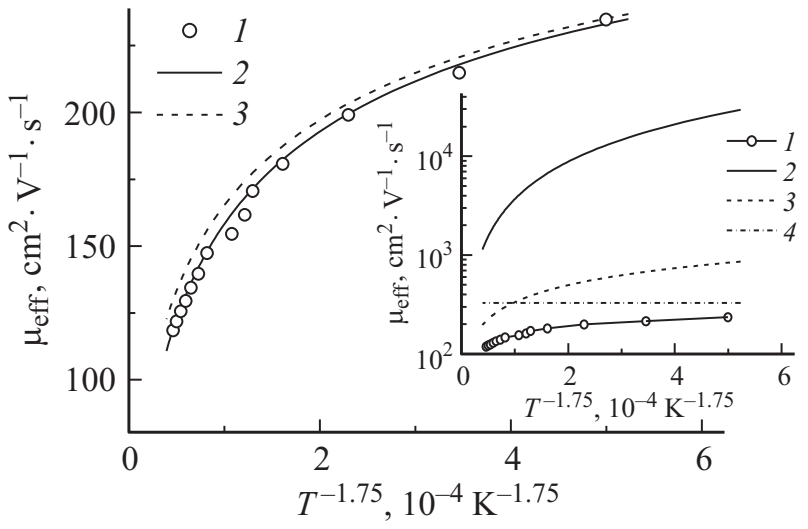

Рис. 5. Зависимости $\mu_{\mathrm{eff}}\left(T^{-1.75}\right)$, полученные экспериментально в режиме $3 A(1)$ и при использовании выражения (5) с учетом $\mu_{V p h}(2)$ и без учета $\mu_{V p h}(3)$. На вставке: экспериментальные данные $(1)$ и используемые при аппроксимации (5) компоненты: $\mu_{V p h}(2), \mu_{s p h}(3), \mu_{s r}(4)$.

области 3 выражение (3) можно записать в виде

$$
\begin{aligned}
\mu_{\mathrm{eff}}(T) & =\left(+\frac{1}{\mu_{V p h}(T)}+\frac{1}{\mu_{s p h}(T)}+\frac{1}{\mu_{s r}(T)}\right)^{-1} \\
& =\left(\frac{1}{\mu_{0}(T / 300)^{-2.3}}+\frac{1}{b T^{-1}}+\frac{1}{\mu_{s r}}\right)^{-1} .
\end{aligned}
$$

Поскольку с изменением температуры происходит изменение наклона зависимостей $\mu_{\mathrm{eff}}\left(T^{-1.75}\right.$ ) (рис. $\left.4, b\right)$, т. е. изменяется доминирующий механизм рассеяния носителей заряда, то аппроксимация температурной зависимости подвижности выражением (5) в режиме $3 A$ позволяет уверенно выделить компоненты подвижности, ограниченные рассеянием на поверхностных фононах и микрорельефе гетерограницы.

На рис. 5 представлена зависимость $\mu_{\mathrm{eff}}\left(T^{-1.75}\right)$, полученная экспериментально в режиме $3 A$, и ее аппроксимации, полученные при использовании выражения (5) без учета и с учетом рассеяния на объемных фононах. На рис. 5 показаны также температурные зависимости компонентов подвижности, используемых при аппроксимации по формуле (5). Видно, что для исследуемых пленок в режиме $3 A$ значения $\mu_{s r}$ составляют $327 \mathrm{~cm}^{2} \cdot \mathrm{B}^{-1} \cdot \mathrm{c}^{-1}$, значения $\mu_{s p h}$ варьируются в диапазоне $200-860 \mathrm{~cm}^{2} \cdot \mathrm{B}^{-1} \cdot \mathrm{c}^{-1}$ и подчиняются закону $\mu_{s p h}=6.45 \cdot 10^{4} \mathrm{~T}^{-1}$ при изменении температуры от 300 до $78 \mathrm{~K}$.

Зависимости $\mu_{\text {eff }}\left(T^{-1.75}\right)$, полученные при использовании выражения (5) для пленки КНИ в режимах $3 B$ и $3 C$, показаны на рис. $4, b$. Видно, что выражение (5) хорошо описывает температурную зависимость $\mu_{\mathrm{eff}}\left(T^{-1.75}\right)$ при любом состоянии пленки со стороны TG в интервале высоких значений $N_{e}$. В режимах $3 C$ (условиях инверсии пленки КНИ со стороны ТG) были получены следующие значения для компонентов подвижности: $\mu_{s r}=432 \mathrm{~cm}^{2} \cdot \mathrm{B}^{-1} \cdot \mathrm{c}^{-1}, \mu_{s p h}$ варьируются в диа- 
пазоне $264-1135 \mathrm{~cm}^{2} \cdot \mathrm{B}^{-1} \cdot \mathrm{c}^{-1}$ и подчиняются закону $\mu_{s p h}=8.5 \cdot 10^{4} T^{-1}$ при изменении температуры от 300 до $78 \mathrm{~K}$.

Следует отметить, что в интервале средних значений $N_{e}$ используемое приближение $\mu_{s p h}(T)=b T^{-1}$ дает уже существенные отклонения аппроксимации $\mu_{\mathrm{eff}}(T)$ от экспериментальных данных (рис. 4, $a$ ).

Анализ зависимостей $\mu_{\mathrm{eff}}\left(T^{-1.75}\right)$ показал, что в интервале 3 , при больших значениях $\left.N_{e}: 1\right)$ вклад $\mu_{V p h}$ в эффективную подвижность электронов, действительно, несуществен и не превышает $8 \%$ (рис. 5), 2) степенной показатель составляет -1.75 и изменение наклона $B_{i}$ температурных зависимостей $\mu_{\text {eff }}$ определяется постоянной составляющей $\mu_{s r}$ при зависимостях $\mu_{V p h} \propto T^{-2.5}$ и $\mu_{s p h} \propto T^{-1}$ для других компонентов $\left.\mu_{\mathrm{eff}}, 3\right)$ для исследуемых пленок КНИ в области температур, близких к комнатной, доминирует рассеяние на поверхностных фононах.

Действительно, из рис. 5 (вставка) видно, что в области низких температур значение $\mu_{s r}$ меньше $\mu_{s p h}$ и $\mu_{V p h}$, т.е. подвижность ограничена рассеянием на микрорельефе ГР. Рассеяние на микрорельефе ГР является температурно-независимым, что и объясняет параллельный сдвиг зависимостей $\mu_{\mathrm{eff}}\left(T^{-1.75}\right)$ по оси $\mu_{\mathrm{eff}}$ при температурах $T<180 \mathrm{~K}$ (рис. $4, b$ ). Напротив, в области температур, близких к комнатной, даже в режиме $3 A$, когда электроны максимально смещены к ГР пленка/BOX, значения $\mu_{s p h}$ меньше $\mu_{s r}$, доминирует рассеяние на поверхностных фононах. Таким образом, в исследуемых пленках КНИ в области не только средних, но и больших значений $N_{e}$ при температурах, близких к комнатной, доминирует рассеяние на фононах. Вклад рассеяния на микрорельефе ГР изменяет наклон зависимостей $\mu_{\mathrm{eff}}\left(T^{-1.75}\right)$. Однако доминирующим этот механизм становится только в области низких температур $(<180 \mathrm{~K})$ и высокой плотности индуцированных носителей заряда $\left(N_{e}>10^{12} \mathrm{~cm}^{-2}\right)$.

\section{5. Заключение}

В работе исследованы зависимости подвижности электронов $\mu_{\text {eff }}$ от их плотности $N_{e}$ в инверсионных слоях полностью обедняемых КНИ МОП транзисторов и температурные зависимости $\mu_{\text {eff }}$ при разных режимах (инверсия-обогащение) пленки КНИ со стороны одной из гетерограниц.

Установлено, что при $T=300 \mathrm{~K}$ в исследуемых пленках КНИ подвижность электронов изменяется в диапазоне 690-110 $\mathrm{cm}^{2} \mathrm{~B}^{-1} \mathrm{c}^{-1}$ при изменении $N_{e}$ в пределах $2 \cdot 10^{11}-1.2 \cdot 10^{13} \mathrm{~cm}^{-2}$. Показано, что зависимости $\mu_{\mathrm{eff}}\left(N_{e}\right)$ для электронов в инверсионных слоях, как и в слоях обогащения в полностью обедняемых КНИ транзисторах, могут быть аппроксимированы степенными функциями $\propto N_{e}^{-n}$. Показатель $n$ принимает постоянные значения в области низких и высоких значений $N_{e}: n=0$ при $N_{e}<1 \cdot 10^{12} \mathrm{~cm}^{-2}$ и $n=0.7$ при $N_{e}>6 \cdot 10^{12} \mathrm{~cm}^{-2}$.
В области средних значений $N_{e}$ показатель $n$ изменяется в диапазоне 0.1-0.41 при изменении состояния поверхности пленки от обогащения до инверсии и принимает значения $\sim 0.3$ при условии обеднения слоя КНИ.

Показано, что температурные зависимости подвижности в области средних и высоких значений $N_{e}$ подчиняются закону $\propto T^{-1.75}$ с двумя характерными наклонами в диапазонах $\sim(300-180)$ и $\sim(180-78)$ К. Изменение наклона зависимостей $\mu_{\mathrm{eff}}\left(T^{-1.75}\right)$ определяется изменением доминирующего механизма рассеяния.

Выделены компоненты подвижности электронов, обусловленные их рассеянием на поверхностных фононах и на микрорельефе границы раздела пленка/скрытый диэлектрик структур КНИ. Определено, что при температурах, близких к комнатной, в области средних и высоких значений $N_{e}$ доминирующим механизмом рассеяния электронов является рассеяние на фононах. Рассеяние на микрорельефе границы раздела КНИ/ВОХ доминирует в области низких температур при высокой плотности индуцированных носителей заряда независимо от режима поверхности пленки.

\section{Список литературы}

[1] T. Rudenko, V. Kilchytska, S. Burignat, J.P. Raskin, F. Andrieu, O. Faynot. Sol. St. Electron., 54, 164 (2010).

[2] A. Ohata, S. Cristoloveanu, A. Vandooren, M. Casse, F. Dauge. Microelectron. Eng., 80, 245 (2005).

[3] E. Simoen, A. Mercha, C. Claeys, N. Lukyanchikova, N. Garbar. IEEE Trans. Electron Dev., 51 (6), 1008 (2004).

[4] S. Eminente, S. Cristoloveanu, R. Clerc, A. Ohata, G. Ghibaudo. Sol. St. Electron., 51, 239 (2007).

[5] C. Lombardi, S. Manzini, A. Saporito, M. Vanzi. IEEE Trans. Computer-Aided Design, 7 (11), 1164 (1988).

[6] M.N. Darwish, J.L. Lentz, M.R. Pinto, P.M. Zeitzoff, T.J. Krutsick, H.H. Vuong. IEEE Trans. Electron Dev., 44 (9), 1529 (1997).

[7] A. Ciprut, A. Chelly, A. Karsenty. Active Passive Electron. Comp., 2015, 1 (2015).

[8] S. Takagi, A. Toriumi, M. Iwase, H. Tango. IEEE Trans. Electron Dev., 41 (12), 2357 (1994).

[9] K. Chen, H. Clement Wann, J. Dunster, P.K. Ko, C. Hu. Sol. St. Electron., 39 (10), 1515 (1996).

[12] Г.Я. Красников. Конструктивно-технологические особенности субмикронных МОП-транзисторов (М., Техносфера, 2002).

[11] S. Cristoloveanu, N. Rodriguez, F. Gamiz. IEEE Trans. Electron Dev., 57 (6), 1327 (2010).

[10] О.В. Наумова, Э.Г. Зайцева, Б.И. Фомин, М.А. Ильницкий, В.П. Попов. ФТП, 49 (10), 1360 (2015).

[13] O.V. Naumova, B.I. Fomin, D.A. Nasimov, N.V. Dudchenko, S.F. Devyatova, E.D. Zhanaev, V.P. Popov, A.V. Latyshev, A.L. Aseev, Yu.D. Ivanov, A.I. Archakov. Semocond. Sci. Technol., 25, 055004 (2010).

[14] K. Uchida, J. Koga, S. Takagi. J. Appl. Phys., 102, 074510 (2007).

[15] A. Ortiz-Conde, F.J. Garcia Sanchez, J.J. Liou, A. Cerdeira, M. Estrada, Y. Yue. Microelectron. Reliab., 42, 583 (2002). 
[16] H.K. Lim, J.G. Fossum. IEEE Trans. Electron Dev., 30 (10), 1244 (1983).

[17] T. Rudenko, A. Nazarov, V. Kilchytska, D. Flandre. Sol. St. Electron., 117, 66 (2016).

[18] D.B. Klaassen. Sol. St. Electron., 35 (7), 953 (1992).

[19] D.B. Klaassen. Sol. St. Electron., 35 (7), 961 (1992).

[20] N. Goel, A. Tripathi. Intern. J. Computer Applications, 42 (21), 56 (2012).

[21] N. Rodriguez, J.B. Roldan, F. Gamiz. Semicond. Sci. Technol., 22, 348 (2007).

[22] A. Chaudhry, S. Sangwan, J. N. Roy. Contemp. Eng. Sci., 4 (5), 229 (2011).

Редактор Л.В. Шаронова

\section{Electron mobility in inversion layers of fully depleted SOI films}

\section{E.G. Zaitseva, O.V. Naumova, B.I. Fomin}

Rzhanov Institute of Semiconductor Physics, Siberian Branch of Russian Academy of Sciences, 630090 Novosibirsk, Russia

Abstract Dependences of the electron mobility $\mu_{\text {eff }}$ on the density of induced charge carriers $N_{e}$ and on the temperature $T$ for different SOI film states (inversion-accumulation) near one of its surface in the inversion layers of fully depleted doublegate silicon-on-insulator (SOI) metal-oxide-semiconductor fieldeffect transistors (MOSFET) are investigated. It is shown that the $\mu_{\text {eff }}(T)$ dependences allow to reveal the mobility components limited by surface phonons scattering and by surface roughness scattering at high density of charge carriers $\left(N_{e}>6 \cdot 10^{12} \mathrm{~cm}^{-2}\right)$. The $\mu_{\text {eff }}\left(N_{e}\right)$ dependences can be approximated by power functions $\mu_{\text {eff }}\left(N_{e}\right) \propto N_{e}^{-n}$. The values of the exponent $n$ in the dependences and the dominant scattering mechanisms of electrons induced near the interface of SOI film with buried oxide for various range of $N_{e}$ and for various film states near the surface are determined. 